\section{Creep of Anisotropic Clay: Microplane Model}

\section{By Zdeněk P. Bažant, ${ }^{1}$ F. ASCE and Jin-Keun Kim, ${ }^{2}$ M. ASCE}

ABstaACT: Undrained constant-volume creep of anisotropically consolidated specimens of clay is mathematically described by the microplane model, which is based on the assumption that the shear strain rates on the contact planes between mutually sliding clay platelets (the microplanes) are the resolved components of the macroscopic strain rate. Thus, the microstructure is assumed to be kinematically constrained. The rate of shear on the microplanes is assumed to be governed by activation energy (rate process theory). The matrix of the current viscosities is obtained as an integral over all spatial directions involving the shear strain rates for the microplanes. This integral, which is evaluated numerically as a summation, gives the dependence of the viscosity matrix on the applied macroscopic stress. Anisotropy of the clay is characterized by a function of the spherical angles describing the relative frequency of clay plate-
lets of various orientations. This function can be approximately estimated from lets of various orientations. This function can be approximately estimated fram eters for the stress dependence and one for the time decay of creep rate. Satisfactory fits of test data on remolded clay samples anisotropically consolidated in the laboratory are achieved, but applicability in the field remains experimentally unverified.

\section{INTRODUCTION}

It is now widely recognized that formulation of a realistic and broadly applicable constitutive relation for clay, as well as any other materials, has to be based on micromechanics of deformation and description of the physical processes involved on the microstructural level. The physical concept, which describes the essence of creep in many materials including clay, is the concept of activation energy, as introduced in the rate-process theory by Eyring and coworkers $(11,14)$. That this concept applies to clay was established by Murayama and Shibata $(19,20,21)$, Mitchell, et al. $(17,18,25)$, Christensen and $W u(10)$, and others $(9,30,31)$. However, the application of the activation energy concept in these formulations was restricted to a relation between a single stress component and a single strain component, implying that the stress level for all interparticle contacts in which sliding occurs was the same. Thus, the details of the microstructure, and the triaxial tensorial aspects of deformation were ignored, for the sake of simplicity. This approach, therefore, could not distinguish different shearing rates on planes of various orientations with different shear stresses, and it could not take into account anisotropy, which is typical of most clays.

The tensorial aspect and micromechanics of deformation based on microstructure geometry were introduced into an activation energy formulation for clay in Ref. 8. This work modeled, in an idealized way,

'Prof. of Civ. Engrg. and Dir., Center for Concrete and Geomaterials, Northwestern Univ., Evanston, IL 60201.

${ }^{2}$ Grad. Research Asst., Northwestern Univ.; presently Asst. Prof., Dept. of Civ. Engrg., Korea Advanced Inst. of Sci. and Tech., Seoul, Korea.

Note--Discussion open until September 1, 1986. To extend the closing date one month, a written request must be filed with the ASCE Manager of Journals. The manuscript for this paper was submitted for review and possible publication on October 11, 1984. This paper is part of the Journal of Geotechnical Engineering, Vol. 112, No. 4, April, 1986. CASCE, ISSN 0733-9410/86/0004-0458/\$01.00. Paper No. 20557. the fact that clays normally possess a fabric with a preferred particle orientation causing the creep properties to be anisotropic. A two-dimensional microstructural model was set up to take into consideration the distribution of particle orientations. The model was based on a triangular cell of three straight-line particles sliding over each other at a speed determined by activation energy according to the shear force transmitted by the interparticle contact. By equating the rate of energy dissipation within all such cells to the macroscopic continuum energy dissipation rate, and using variational calculus arguments, the curren viscosity matrix and the matrix of nonviscous stress components was derived and their dependence on a two-dimensional stress tensor was determined. The anisotropic viscosity matrix and nonviscous stress matrix were obtained by averaging their values for all possible triangula cells according to a given frequency distribution of particle orientations. Although three-dimensional creep was approximately described through a combination of two-dimensional models for coordinate planes, the conceptual limitation of the approach from Ref. 8 to two-dimensional deformation has been a serious drawback. The triangular cell might be generalized to three dimensions as a polyhedral cell, however the formulation would become rather complicated.

Therefore, a different approach is adopted in this study. Instead of dealing with triangular cells in which each particle slides over its neighbors, we will deal directly with the individual contacts assuming that their relative slip rate is determined by the macroscopic strain rate tensor in a manner analogous to the well-known slip theory of plasticity (3) but differs from it by the use of a kinematical instead of statical constraint. In contrast to the triangular cell model from Ref. 8, this approach does not imply that every clay particle slips over its neighbor. Rather, it permits that the clay particles form groups and relative slips occur only between particles from neighboring groups but not between the particles of the same group. This picture is more realistic according to the current knowledge of clay microstructure.

Our ultimate objective is to formulate the current incremental viscosity matrix as a function of the macroscopic strain rate tensor or stress tensor, and to compare the formulation to existing laboratory data on the anisotropy of creep. Our analysis will be limited to deviatoric creep. Vol: ume dilatancy will be ignored since its treatment is beyond the scope of present work. Development of a refined model that covers dilatancy is in progress and is planned for a subsequent paper.

\section{Objectives and Method of Approach}

We are trying to develop a constitutive model of the following properties:

1. The inelastic deformation is caused by sliding at interparticle contacts.

2. This sliding is governed by activation energy (rate process theory).

3. The frequency distribution of the orientations of the interparticle contact planes is prescribed, e.g., according to microstructure observations. 
4. The model is not restricted to two dimensions but takes into account a three-dimensional frequency distribution of the contact plane orientations.

5. The model predicts the current incremental viscosity matrix as a function of the current strain rate tensor or stress tensor.

6 . The model can be implemented in a finite element computer program.

To correlate interparticle sliding to the macroscopic strain rate tensor, we adopt an approach analogous to the slip theory of plasticity (3). In this approach, first suggested by Taylor (28), the constitutive properties are defined independently on planes of various orientations within the material, and the contributions from all such planes are then suitably superimposed. As suggested by Taylor, there are two simple possibilities; either the microstructure is statically constrained, in which case the stresses (on each plane contributing to inelastic deformation) are the resolved components of the macroscopic stress tensor, or the microstructure is kinematically constrained, in which case the strains or strain rates are the resolved components of the macroscopic tensor of strain or strain rate. The former approach has been used in all works dealing with plasticity of metals, and was also applied to clay by Pande and Sharma (22), although without recourse to the activation energy concept. The latter approach, i.e., a kinematically constrained microstructure, was introduced in a recent study $(5,7)$ of the micromechanics of inelastic deformation in concrete. This approach was adopted for two reasons: (1) It was necessary to model strain-softening, which does not permit a statically constrained microstructure for reasons of instability; and (2) a better agreement with test data was obtained, apparently because the highly localized inelastic deformation in the contact zones between aggregate particles is closer to being constrained to the macroscopic deformation rather than to the macroscopic stress. This latter reason seems to also apply to clay, and we will therefore assume a kinematically constrained microstructure even though the aforementioned first reason is not applicable here since we do not attempt to model strain softening in clay. Also, the second approach is more stable numerically.

The planes of various orientations in the microstructure in which the inelastic deformation is concentrated may be concisely called the microplanes $(4,5,7)$. In previous works, the kinematically constrained microplane model, which was developed for concrete $(5,7)$, involved only normal stresses and strains on the microplanes, while the classical statically constrained models for polycrystalline metals involved only shear stresses and strains. For clay, we will use the shear stresses and shear strain rates on the microplanes in order to describe the inelastic deformation because particle slipping is a shear process (however, particle slipping possibly caused by volume change must be ignored in this approach).

Under a kinematic constraint, the general formulation of the microplane model developed in Ref. 4 will be followed here. However, the normal strain rates on the microplanes, which were used in Ref. 4, may be neglected since we intend to describe only the deviatoric deformations and relegate the description of volume changes to subsequent work.

The basic assumption in the present model is that the sole source of inelastic deformation (creep) of clay is interparticle sliding. This means that we neglect a possible contribution from rearrangements of platelet connectivity due to locally large relative displacements and rotations.

\section{Derivation of Viscosity Tensor}

First we need to calculate the components $\epsilon_{j}^{n}$ of the strain on a microplane of normal $\overrightarrow{\mathbf{n}}$ at which slip in interparticle contact is taking place [Fig. 1(a)]. We use cartesian tensors in coordinate system $x_{i}(i=1,2,3)$. Latin lower-case subscripts denote components along these axes, and their repetition implies summation from 1 to 3 , as usual. Because a kinematically constrained microstructure is assumed, the components of the strain vector $\overrightarrow{\boldsymbol{\epsilon}}^{n}$ on any microplane are the resolved components of the macroscopic strain tensor $\epsilon_{i j}$, i.e.

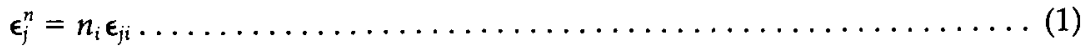

in which $n_{i}=$ the direction cosines of the unit normal to the microplane. Projection of the microplane strain vector (Eq. 1) onto $\overrightarrow{\mathbf{n}}$ yields the following magnitude of the normal strain on the microplane and components of its vector:

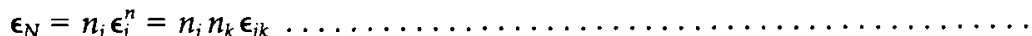

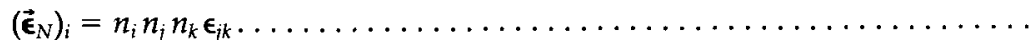

The magnitude of the strain vector on the microplane is

$\left|\vec{\epsilon}^{n}\right|=\left(\epsilon_{j}^{n} \epsilon_{j}^{n}\right)^{1 / 2}=\left(n_{i} \epsilon_{j i} n_{k} \epsilon_{j k}\right)^{1 / 2}$

and the vector of the tangential (shear) strain component on the microplane then is [Fig. 1(a)]

$\overrightarrow{\boldsymbol{\epsilon}}_{T}=\overrightarrow{\boldsymbol{\epsilon}}^{n}-\overrightarrow{\boldsymbol{\epsilon}}_{N}$

the magnitude of which is

$\boldsymbol{\epsilon}_{T}=\left(\left|\overrightarrow{\boldsymbol{\epsilon}}^{n}\right|^{2}-\boldsymbol{\epsilon}_{N}^{2}\right)^{1 / 2}$

or $\epsilon_{T}=\left|\vec{\epsilon}_{T}\right|=\left(\epsilon_{T_{i}} \epsilon_{T}\right)^{1 / 2}$

This leads to the following expressions for the components of the shear

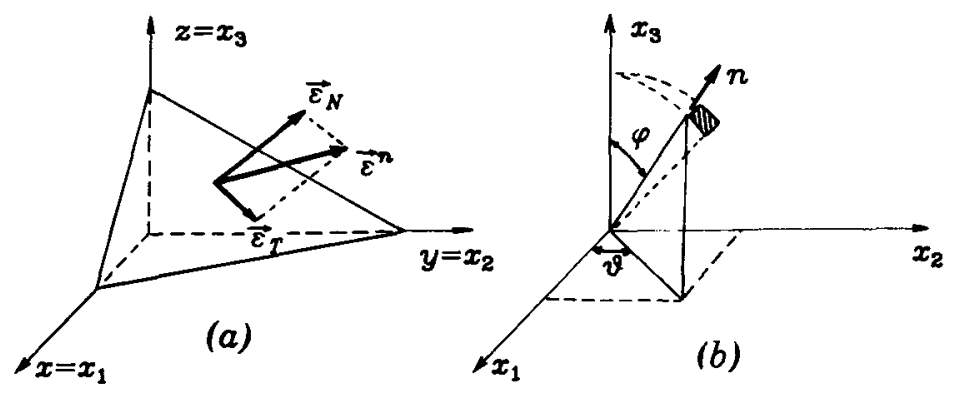

FIG. 1.-Strain Vector Components on: (a) Microplane; and (b) Spherical Coordinate System 


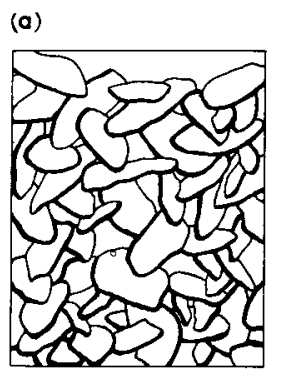

(b)
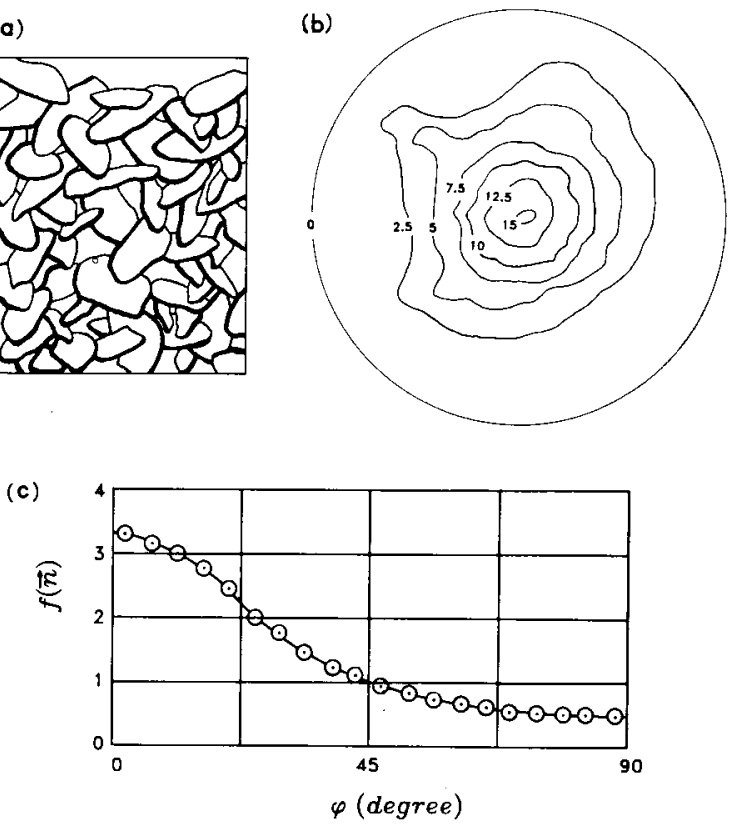

FIG. 2.-(a) Idealized Microstructure of Clay; (b) Contours of Equal Frequency of Angle of Inclination of Clay Platelet Normal with Direction of Maximum Principal Consolidation Stress; (c) Frequency Distribution Function of Platelet Orientations (from Test Data of Ref. 16, Obtained by X-ray Scattering)

strain vector on the microplane and for the magnitude of this vector:

$\epsilon_{T_{i}}=\left(n_{k} \delta_{i j}-n_{i} n_{j} n_{k}\right) \epsilon_{j k} \ldots \ldots \ldots \ldots \ldots \ldots \ldots \ldots \ldots \ldots \ldots \ldots \ldots \ldots \ldots \ldots$

$\boldsymbol{\epsilon}_{T}=\left[n_{i} \epsilon_{j k} n_{k}\left(\epsilon_{j k}-n_{j} n_{m} \epsilon_{k m}\right)\right]^{1 / 2}$

The microstructure of clay consists of an agglomerate of rando......... (9) ented microscopic platelets of clay minerals, resembling a house of cards $(1,2,12,13,15,16,27,29)$ [see Fig. 2(a)]. The clay platelets are bound together at isolated contact points by electrostatic forces. These bound are meta-stable, chiefly due to the hydrophylic nature of clay platelets and the presence of water filling all interstitial space. The relative sliding of clay platelets at their contact points is caused by ruptures and reformations of the interparticle bonds. Because the processes of bond rupture are generally described by the rate-process theory $(11,14)$, it is not surprising that this theory has been found applicable to clays $(8,10,17$ $21,25,30,31)$. According to this theory, the rate of sliding in clay platelet contacts, characterized by $\dot{\boldsymbol{\epsilon}}_{T}$ (with the superior dot denoting the time rate) is expressed as

$\dot{\boldsymbol{\epsilon}}_{T}=k_{1} \sinh \left(k_{2} \tau_{T}\right)$

in which $\tau_{T}=$ the, shear (tangential) stress component on the same microplane (i.e., the plane of sliding), and $k_{1}=2 A \frac{k T}{h} t^{-m} e^{-Q / R T}=k_{0} t^{-m}$

$k_{2}=\frac{V_{a}}{R T}$

Here, $T=$ absolute temperature; $Q=$ activation energy of interparticle bonds = activation energy of creep; $R=$ universal gas constant; $k=$ Boltzman constant; $h=$ Planck constant; $V_{a}=$ activation volume; $m, A$ = empirical constants of which $A$ characterizes the number of active bonds at the moment of stress application; and the power function $t^{-m}$ of time $t$ describes, according to Singh and Mitchell (25), the time decay of the creep rate at constant stress caused by gradual exhaustion of the active bond sites. For our purposes, it is more convenient to use the inverted form of Eq. 10, which is

$\tau_{T}=\frac{1}{k_{2}} \sinh ^{-1} \frac{\dot{\epsilon}_{T}}{k_{1}}$

According to the current view of creep mechanism, groups of clay platelets (particles) tend to be essentially fixed and move together, while the relative sliding occurs only between particles from adjacent groups but not within those from one and the same group. This mechanism of creep requires no change in our formulation since Eqs. 10-13 apply only to the active interparticle contacts between these groups of particles and not to the fixed interparticle contacts within the groups.

The magnitude $\tau_{T}$ has already been calculated but its direction must still be determined. The clay platelets have a crystalline structure, and so, no doubt, there exist some preferred directions in the plane of each platelet. Thus, the slip direction would not, in general, coincide with the shear stress direction in the contact plane. However, the available measurement techniques, such as the $\mathrm{X}$-ray diffraction, reveal nothing about preferred orientations with the plane of clay platelet; at best they give information only on the frequency of average orientations of the normals to the platelets. From the statistical viewpoint, we must assume that, when viewed from the tip of vector $\vec{n}$, the deviations of the slip directions to the left and to the right of the shear stress vector direction are equally likely (frequent) among all contact planes of orientation $\overrightarrow{\mathbf{n}}$. Therefore, we will assume that, as an approximation, the slip direction in each contact plane on the average coincides with the shear stress direction, i.e., vectors $\dot{\boldsymbol{\epsilon}}_{T}$ and $\overrightarrow{\boldsymbol{\tau}}_{T}$ are in each microplane parallel. This means that the components of the shear stress vector are

$\tau_{T_{i}}=\frac{1}{k_{2}} e_{i} \sinh ^{-1} \frac{\dot{\epsilon}_{T}}{k_{1}}$

in which $e_{i}=$ the direction cosines of the shear strain rate vector, i.e. $e_{i}=\frac{\dot{\boldsymbol{\epsilon}}_{T_{i}}}{\dot{\boldsymbol{\epsilon}}_{T}}$.

Parallel vectors of slip and shear stress on each microplane are also obtained (4) from a formulation analogous to the theory of plasticity, in 
which the slip direction is assumed to be normal to a two-dimensional plastic (or viscoplastic) potential surface in the plane of each contact, provided this potential surface is isotropic (i.e., circular) within each contact plane. The assumption of isotropy within each contact plane (albeit not for clay as a whole) is again inevitable if there is no information on preferred orientations within the plane of each platelet. Note also that a theory in which the slip and shear stress directions in each microplane need not be parallel would be much more complicated than the present one.

In contrast to the previous formulation from Ref. 8 , the present model does not require that every inter-platelet contact must be tip to plane. Contacts edge to plane, or tip to edge, or edge to edge are also admissible in these dimensions since they permit sliding. However, the sliding contacts can hardly be imagined as tip to tip, since there would be no room for finite relative displacement. Plane to plane contacts of platelets, giving the densest possible configuration of solids, are unlikely to occur.

To establish the equilibrium relation between the microstresses on the microplanes of all orientations and the macroscopic stress tensor, we may use the principle of virtual work. The macroscopic virtual work of stresses per unit volume of clay is $\sigma_{i j} \delta \dot{\epsilon}_{i j}$, in which the macroscopic stress tensor $\sigma_{i j}$ must be interpreted as the effective stress tensor, i.e.

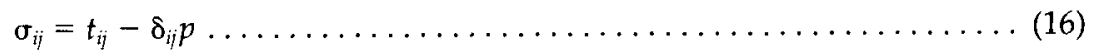

in which $p=$ pore water pressure; and $t_{i j}=$ total stress in the solid-water system. The principle of virtual work requires that the virtual work of the macroscopic stresses on any variations $\delta \dot{\epsilon}_{i j}$ of the macroscopic strain rate $\dot{\epsilon}_{i j}$ within a small unit sphere (of radius 1) must be equal to the virtual work done by the shear stresses on the microplanes of all orientations. This condition may be written as

$$
\begin{aligned}
& \delta \dot{W}=\frac{4 \pi}{3} \sigma_{i j} \delta \dot{\epsilon}_{i j}=2 \int_{S} \tau_{T_{r}} \delta \dot{\epsilon}_{T_{r}} f(\overrightarrow{\mathbf{n}}) d S \\
& =2 \int_{S} \frac{1}{k_{2} \dot{\epsilon}_{T}}\left(n_{m} \delta_{k r}-n_{r} n_{k} n_{m}\right) \dot{\epsilon}_{k m}\left(\sinh ^{-1} \frac{\dot{\epsilon}_{T}}{k_{1}}\right)\left(n_{j} \delta_{i r}-n_{r} n_{i} n_{j}\right) \delta \dot{\epsilon}_{i j} f(\overrightarrow{\mathbf{n}}) d S \ldots \\
& \text { or } \sigma_{i j} \delta \dot{\epsilon}_{i j}=\left[\frac{3}{2 \pi} \int_{S} b_{i j k m}^{\prime} \frac{1}{k_{2} \dot{\epsilon}_{T}}\left(\sinh ^{-1} \frac{\dot{\epsilon}_{T}}{k_{1}}\right) f(\overrightarrow{\mathbf{n}}) d S\right] \dot{\epsilon}_{k m} \delta \dot{\epsilon}_{i j} \ldots \ldots \ldots
\end{aligned}
$$

The integration needs to be carried out only over the surface of a unit hemisphere, $S$, since the integrand values at two diametrically opposite points of the surface of the sphere are equal. $f(\overrightarrow{\mathbf{n}})$ is the given distribution function for the frequency of orientations of the planes of sliding interplatelet contacts. Furthermore

$b_{i j k m}^{\prime}=\left(n_{j} \delta_{i r}-n_{r} n_{i} n_{j}\right)\left(n_{m} \delta_{k r}-n_{r} n_{k} n_{m}\right)=\delta_{i k} n_{j} n_{m}-n_{i} n_{j} n_{k} n_{m} \ldots \ldots$ (19)

This tensor is not symmetric with regard to the interchange of subscript $i$ with $j$ and $k$ with $m$. But it may be written as a sum of a symmetric part and an antisymmetric part

$b_{i j k m}^{\prime}=b_{i j k m}+\tilde{b}_{i j k m}$ in which the symmetric part is

$b_{i j k m}=\frac{1}{4}\left(\delta_{i k} n_{j} n_{m}+\delta_{j k} n_{i} n_{m}+\delta_{i m} n_{j} n_{k}+\delta_{j m} n_{i} n_{k}\right)-n_{i} n_{j} n_{k} n_{m} \ldots \ldots \ldots$

Therefore, it is always true that

$\tilde{b}_{i j k m} \dot{\epsilon}_{k m} \delta \dot{\epsilon}_{i j}=0$

because tensors $\dot{\epsilon}_{k m}$ and $\delta \dot{\epsilon}_{i j}$ are symmetric. Now, substituting Eq. 20 into Eq. 18 and taking into account Eq. 22, we find that

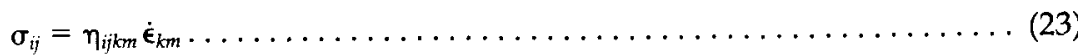

in which $\eta_{i j k m}$ represents the fourth-order tensor of current viscosities, defined as

$\eta_{i j k m}=\frac{3}{2 \pi} \int_{S} b_{i j k m} \frac{1}{k_{2} \dot{\epsilon}_{T}}\left(\sinh ^{-1} \frac{\dot{\epsilon}_{T}}{k_{\mathbf{1}}}\right) f(\overrightarrow{\mathbf{n}}) d S$

Eqs. 23 or 26 , with the viscosity tensor given by Eqs. 24 and 21, represents the desired stress-strain relation which may be used in structural analysis.

It should be realized that the foregoing stress-strain relation does not involve volumetric stress and strain components and leaves them indeterminate. Indeed, the macroscopic volumetric stress $\sigma^{V}$ causes no shear stresses $\tau_{T}$ on the microplanes, and therefore its work rate is zero. Likewise, the shear strain rates $\dot{\epsilon}_{T}$ on the microplanes cause no macroscopic change of volume. Therefore, the stress-strain relation in Eq. 23 remains valid if $\sigma_{i j}$ and $\dot{\epsilon}_{k m}$ are replaced with the macroscopic deviatoric stress tensor $\sigma_{i j}^{D}$ and the macroscopic deviatoric strain rate tensor $\dot{\epsilon}_{k m}^{D}$, i.e.

$\sigma_{i j}^{D}=\eta_{i j k m} \dot{\epsilon}_{k m}^{D}$

Also, Eqs. 8-9 for the shear strain on the microplane remain valid if $\epsilon_{j k}$ is replaced by its deviatoric part $\epsilon_{j k}^{D}$, e.g.

$\dot{\boldsymbol{\epsilon}}_{T}=\left[n_{i} \dot{\boldsymbol{\epsilon}}_{j k}^{D} n_{k}\left(\dot{\boldsymbol{\epsilon}}_{j k}^{D}-n_{j} n_{m} \dot{\boldsymbol{\epsilon}}_{k m}^{D}\right)\right]^{1 / 2}$.

Eqs. 23 or 26 , with the viscosity tensor given by Eqs. 24 and 21, represents the desired stress-strain relation which may be used in structural analysis.

Generalization of the present model to cover volumetric deformations (dilatancy) could be obtained by including normal stresses and strains in the stress-strain relations for the microplane. However, this problem is beyond the scope of the present work and must be left for subsequent study since some difficult questions must be resolved with respect to the implications of the critical state theory.

\section{Numerical Solution and Application}

In practical application, the integral in Eq. 24 has to be evaluated numerically. It may be approximated by a finite sum

$\eta_{i j k m} \simeq \sum_{\alpha=1}^{n} 6 w_{\alpha}\left[b_{i j k m} \frac{1}{k_{2} \dot{\epsilon}_{T}}\left(\sinh ^{-1} \frac{\dot{\epsilon}_{T}}{k_{1}}\right) f(\overrightarrow{\mathbf{n}})\right]_{\alpha}$ 
in which subscripts $\alpha$ refer to the values evaluated at certain numerical integration points on the surface of a unit sphere (i.e., certain characteristic directions); and $w_{\alpha}=$ the coefficients or widths of the numerical integration formula, such that $\Sigma_{\alpha} w_{\alpha}=0.5$ for a hemisphere. Because, in finite element programs for incremental loading, the numerical integration needs to be carried out a great number of times, a highly efficient numerical integration formula is needed. For the slip theory of plasticity, the integration was performed using a rectangular grid in the plane of spherical coordinates $\theta$ and $\phi$. This approach, however, is computationally inefficient because the integration points are crowded computationonly low-degree polynomials are integrated exactly, and the singularity arising from the poles appears to make this kind of integration inaccurate.

The integration points should optimally be distributed over the spherical surface as uniformly as possible. A perfectly uniform subdivision is achieved when the microplanes normal to the $\alpha$ directions correspond to the faces of a regular polyhedron. The most faces are possible with the icosahedron, for which $n=10$ (half the number of faces). Numerical experience, however, shows that 10 points do not yield sufficient accuracy. For a greater number of points, of course, a perfectly uniform spacing of $\alpha$ directions is impossible, but still a point distribution which is as close to uniform as possible is needed for good accuracy.

Various numerical integration formulas with more than 10 integration points are given by Stroud (26). All the present calculations are made with McLaren's formula (26) which has 25 points per hemisphere, is symmetric with regard to the cartesian coordinate planes, and is of degree 11 (i.e., integrates exactly all polynomials up to the 11 th degree) For axisymmetric stress states, the 25-point formula actually involves only 6 points with different integrand values. Further optimal integration formulas are derived in Ref. 6 on the basis of Taylor series expansions. These formulas involve $16,21,33,37$, and 61 points per hemisphere, and are of degrees 9 to 13. The 61-point, 13th degree formula is highly accurate, more than needed for the present purpose. For geotechnical engineering applications, accuracy of the 16 point formula of degree 9 appears sufficient $(5,6)$, too.

The numerical solution of response to given loading may be carried out in a step-by-step fashion. Eq. 25 may be approximated by the incremental relation

$\delta \boldsymbol{\sigma}^{D}=\boldsymbol{\eta} \Delta \dot{\boldsymbol{\epsilon}}^{D}+\Delta \boldsymbol{\eta} \dot{\boldsymbol{\epsilon}}^{D}$

in which $\boldsymbol{\sigma}^{D}$ and $\dot{\boldsymbol{\epsilon}}^{D}=6 \times 1$ column matrices of deviatoric components of stress and of strain rate; $\eta$ is the $6 \times 6$ matrix of current viscosities: and $\Delta$ denotes increments. Eq. 28 may be rewritten as

$\Delta \boldsymbol{\sigma}^{D}=\boldsymbol{\eta} \Delta \dot{\boldsymbol{\epsilon}}^{D}-\Delta \boldsymbol{\sigma}^{D^{\prime \prime}} ; \quad \Delta \boldsymbol{\sigma}^{D^{\prime \prime}}=-\Delta \boldsymbol{\eta} \dot{\boldsymbol{\epsilon}}^{D}$

which may be treated as an elastic stress-strain relation if the strain rate increments are treated as strain increments and the viscosity matrix $\eta$ is treated as the elastic moduli matrix; $\Delta \boldsymbol{\sigma}^{D^{\prime \prime}}=$ the column matrix of inelastic stress increments.

The response of a homogeneously stressed anisotropically consolidated clay specimen in an undrained creep test at constant volume may be solved using the following step-by-step algorithm. Superscripts $I, F$ and $M$ label the initial and final values for the loading step, and the values at midstep.

1. Input $t, m$ (determined in advanced by regression), $k_{0}, k_{2}, f(\overrightarrow{\mathbf{n}})^{\alpha}$ $w^{\alpha}, n_{i}^{\alpha}, \dot{\epsilon}_{11_{\max }}$ and tolerance value. Evaluate $k_{1}=k_{0} t^{-m}$. Generate coefficients $b_{i j k m}^{\alpha}$ for all $\alpha$. Initialize $\dot{\boldsymbol{\epsilon}}^{l}=\boldsymbol{\sigma}^{l}=\Delta \boldsymbol{\sigma}=\Delta \hat{\boldsymbol{\sigma}}=\Delta \dot{\boldsymbol{\epsilon}}=\mathbf{0}$. Then specify increment $\Delta \dot{\epsilon}_{11}(>0)$. For the starting values, assume $\Delta \dot{\epsilon}$ except $\Delta \dot{\epsilon}_{11}$ at zero.

2. Set $\dot{\boldsymbol{\epsilon}}^{F}=\dot{\boldsymbol{\epsilon}}^{I}+\Delta \dot{\boldsymbol{\epsilon}}$ and $\dot{\boldsymbol{\epsilon}}^{M}=\dot{\boldsymbol{\epsilon}}^{I}+\Delta \dot{\boldsymbol{\epsilon}} / 2$. From Eq. 27, calculate $\eta_{i j k}^{M}$ based on $\dot{\boldsymbol{\epsilon}}^{M}$ and $\eta_{i j k m}^{F}$ based on $\dot{\boldsymbol{\epsilon}}^{F}$. Then $\Delta \boldsymbol{\eta}=\boldsymbol{\eta}^{F}-\boldsymbol{\eta}^{I}, \Delta \mathbf{v}^{\prime \prime}=-\Delta \eta \boldsymbol{\epsilon}^{M}$. Thus, $\Delta \boldsymbol{\sigma} \eta^{M} \Delta \dot{\boldsymbol{\epsilon}}-\Delta \boldsymbol{\sigma}^{\prime \prime}$ is a system of six linear equations in which six stress or strain increments are given and six are to be solved. Then apply the conditions characterizing the type of test. Typically, $\Delta \dot{\epsilon}_{11}$ and 5 further conditions, $\Delta \sigma_{22}=\Delta \sigma_{33}, \Delta \sigma_{12}=\Delta \sigma_{23}=\Delta \sigma_{31}=0$ and $\Delta \dot{\epsilon}_{11}+\Delta \dot{\epsilon}_{22}+$ $\Delta \dot{\epsilon}_{33}=0$ (no volume change) are given. Then $\Delta \boldsymbol{\sigma}$ and $\Delta \dot{\boldsymbol{\epsilon}}$ (except $\Delta \dot{\boldsymbol{\epsilon}}_{11}$ ) may be solved from given $\Delta \dot{\epsilon}_{11}$.

3 . Iterate step 2 until the differences between the assumed $\Delta \dot{\boldsymbol{\epsilon}}$ and the solved $\Delta \dot{\boldsymbol{\epsilon}}$ become small enough to meet a given tolerance. Then print $\dot{\boldsymbol{\epsilon}}^{F}, \boldsymbol{\sigma}^{F}$, reset $\dot{\boldsymbol{\epsilon}}^{I} \leftarrow \dot{\boldsymbol{\epsilon}}^{F} \dot{\boldsymbol{\sigma}}^{I} \leftarrow \boldsymbol{\sigma}^{F}$. Then, unless $\dot{\boldsymbol{\epsilon}}_{11}$ exceeds given $\dot{\boldsymbol{\epsilon}}_{11_{\max }}$, go to 2 and start solution of the next loading step.

The foregoing algorithm can be simplified when simulating the creep tests of isotropically consolidated specimens loaded so that the principal applied stresses are parallel or normal to the axis of transverse isotropy.

In fitting test data, the objective is to find the values of two input parameters $k_{0}$ and $k_{2}$ for which the fit is optimum. For this purpose, a number of runs of the program described above need to be made for various values of $k_{0}$ and $k_{2}$. The optimum fit may be sought in a trialand-error fashion or by minimizing the sum of the squares of the deviations from test data as a function of $k_{0}$ and $k_{2}$.

\section{Comparisons with Test Data}

Although the function $f(\overrightarrow{\mathbf{n}})$, characterizing the orientation distribution of the planes of sliding contacts, could in principle be determined by fitting a sufficiently extensive set of deformation measurements for various types of loadings, the analytical model is better supported if the distribution function of the orientations of clay platelets is determined by direct measurements. This task, however, involves considerable difficulties.

Direct measurement of the frequencies of the planes of the sliding contacts in various directions is impossible. What can be measured, at least approximately, is the frequency distribution function $F(\overrightarrow{\mathbf{n}})$ of the orientations of the clay platelets. Although function $f(\overrightarrow{\mathbf{n}})$ need not be the same as function $F(\overrightarrow{\mathbf{n}})$, and probably is not, we may assume it to be approximately the same, i.e., $F(\overrightarrow{\mathbf{n}}) \cong f(\overrightarrow{\mathbf{n}})$. Such an assumption would be exactly true only if, for example, each platelet with at least one slidin contact would be parallel to only one sliding contact plane, in which case there would be a one-to-one correspondence between platelets anc sliding contact planes of the same orientation. In reality, one platele 
$y$ have, e.g., two sliding contacts that are both parallel to this platelet, : none that are parallel to it. Such a configuration destroys the one-toine correspondence. Nevertheless, for a very large number of platelets the errors caused by such configurations are likely to offset each other (e.g., one platelet has two sliding contacts that are parallel and another platelet of the same orientation has two sliding contacts none of which are parallel to the platelet).

Further questions arise with regard to the types of interplatelet contacts. For edge-to-edge, tip-to-plane, and plane-to-plane contacts, the plane of the sliding contact is unambiguous and is parallel to one of the contacting platelets. However, for edge-to-edge and tip-to-edge contacts the sliding contact plane is not unambiguously defined. Although it may still be considered as parallel to one of the platelets, the ambiguity may disturb the equivalence of $f(\overrightarrow{\mathbf{n}})$ and $F(\overrightarrow{\mathbf{n}})$. For the tip-to-tip contacts no sliding with a finite relative displacement is possible.

Assuming that $f(\overrightarrow{\mathbf{n}})$ is approximately the same as the distribution function of the clay platelet orientations, one can estimate this function on the basis of X-ray scattering measurements. Although such measurements are difficult and their interpretation frought with ambiguity, they were nevertheless carried out at Northwestern University by Krizek, Edil, and Chawla $(13,15,16)$. The test specimens were cylinders of diameter $35 \mathrm{~mm}$ and length $40 \mathrm{~mm}$, trimmed from blocks of a dispersed or flocculated hydrite 10 kaolin clay that was consolidated anisotropically and isotropically from a slurry according to a procedure described by Sheeran and Krizek (24), Edil and Krizek (13), Krizek, Edil and Ozaydin (16) and Krizek, Chawla and Edil (15). See these works for a detailed description of the intricate specimen preparation and the testing method. Although again various questions arise, one may deduce it from X-ray scattering data, exemplified by the pole diagram in Fig. $2(b)$ of the diffracted intensity, which is measured by a detector for various sample inclinations and is plotted on the basis of an equal area projection of a spherical surface onto a plane. From Fig. 2(b), the distribution function of particle orientations, $f(\overrightarrow{\mathbf{n}})^{\alpha}$ is obtained as plotted in Fig. 2(c). Fig. 3 shows the orientations of the weakest planes (preferred platelet orientations) in various types of specimens tested and analyzed.

Figs. 4-7 exhibit fits of various creep tests reported in the literature

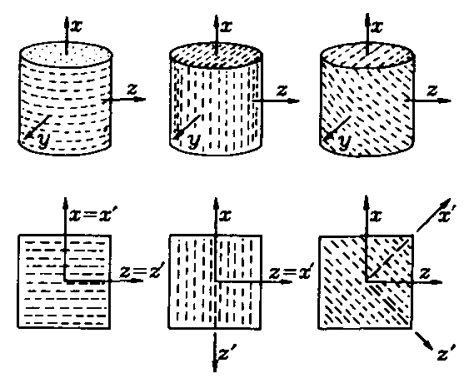

FIG. 3.-Creep Test Specimens Cut at Various Directions with Regard to Preferred Particle Orientation (Indicated by Dashed Lines)

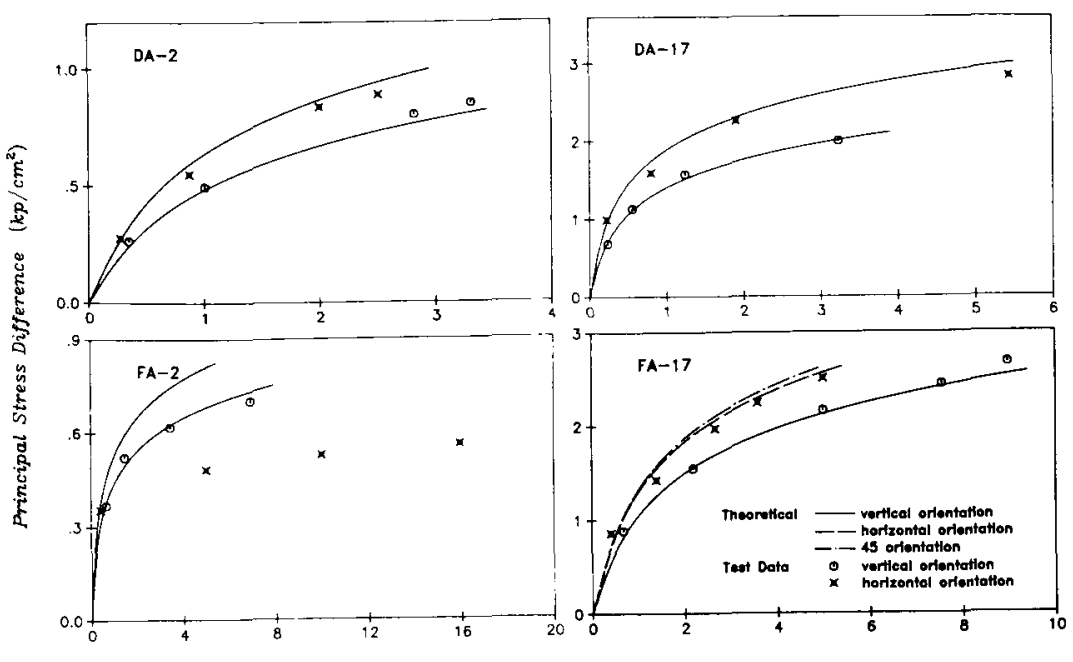

Axial Strain Rate $\left(\times 10^{-8}\right)$

FIG. 4.-Comparison with Test Data for Anlsotropically Consolldated Specimens, Reported by Bažant, Krizek and Ozaydin (1,000 min after Loading): (a) DA-2; (b) DA-17; (c) FA-2; (d) FA-17

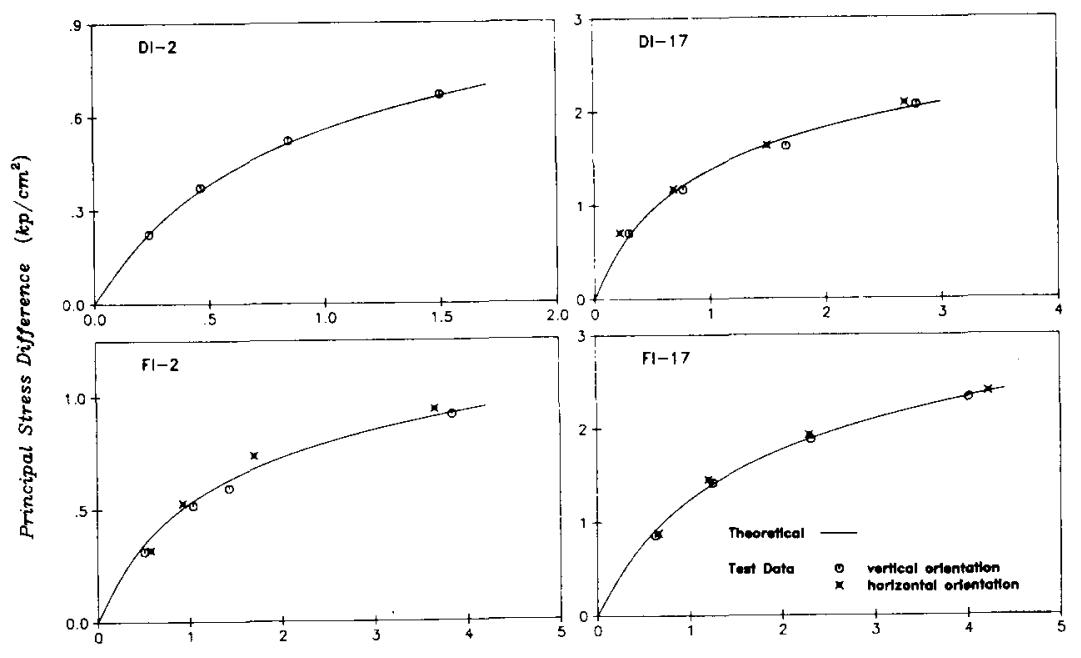

Arial Strain Rate $\left(\times 10^{-6}\right)$

FIG. 5.-Comparison with Test Data for Isotropically Consolidated Specimens Reported by Bažant, Krizek, and Ozaydin (1,000 min after Loading): (a) DI-2; (b) DI-17; (c) FI-2; (d) FI-17 


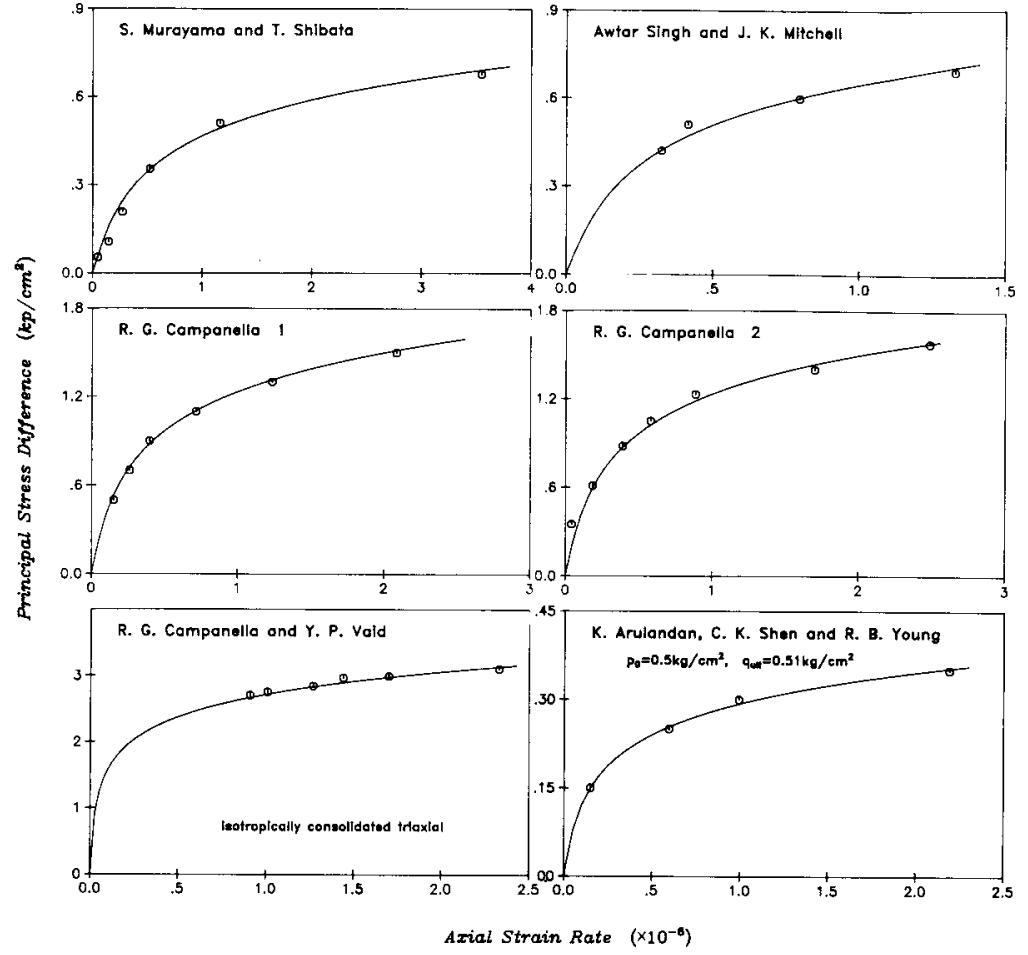

FIG. 6.-Comparison with Test Data for Isotropically Consolidated Specimens Reported by Various Investigators (1 min after Loading)

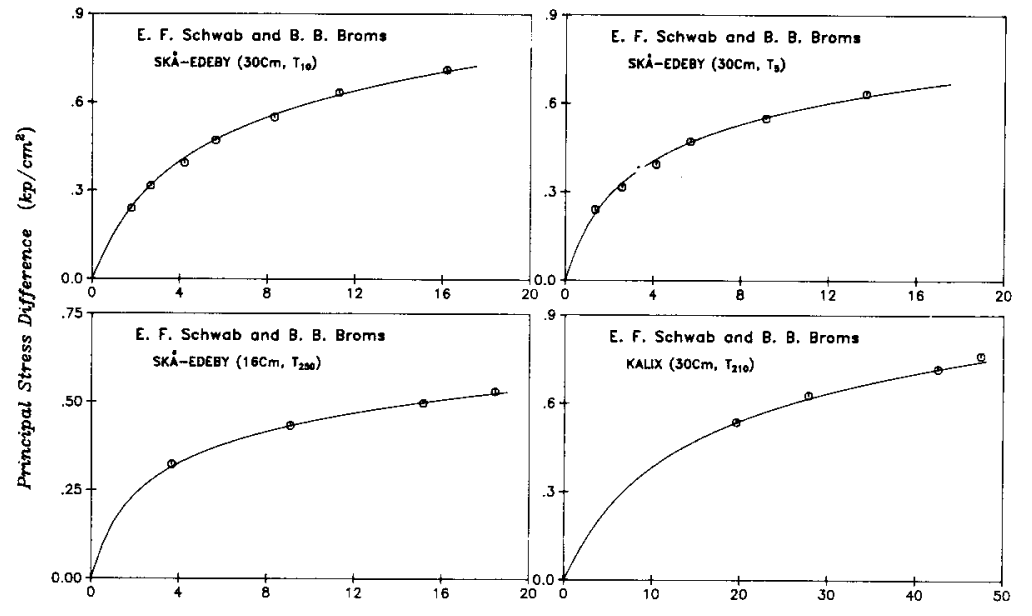

Average Strain Rate $\left(\times 10^{-3}\right)$

FIG. 7.-Comparison with Test Data from Field Vane Tests Reported by Schwab and Broms (1 $\mathrm{min}$ after Loading)
$(1,8,9,18,23)$. In these tests, the clay specimens were prepared using the method developed by Sheeran and Krizek (24), and Krizek, Edil and Ozaydin (16). Fig. 4 represents data for anisotropic clay (8) characterized by the distribution function in Fig. 2, taken from Ref. 16. Two kinds of specimens of the same clay were tested. One kind of specimen was trimmed vertically and the second kind of specimen was trimmed horizontally from each anisotropically (vertically) consolidated specimen. The values of $f(\overrightarrow{\mathbf{n}})^{\alpha}$ for various integration points $\alpha$ are directly read from Fig. 2(c) and are then scaled so that $\Sigma_{\alpha} w_{\alpha} f(\overrightarrow{\mathbf{n}})^{\alpha}$ be 0.5 , exactly. Figs. 5 and 6 exhibit comparisons with test data for isotropically consolidated clays

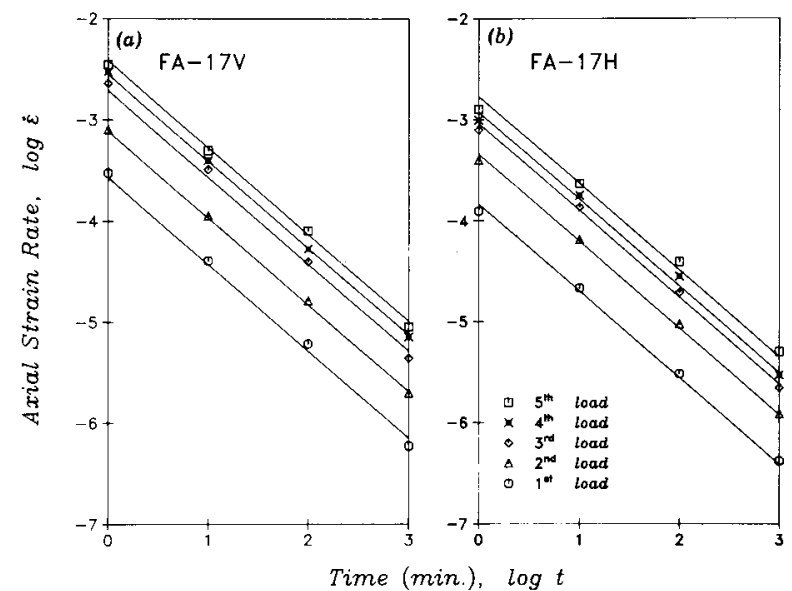

FIG. 8.-Dependence of Strain-Rate on Time under Constant Load: (a) FA-17V (b) FA-17H
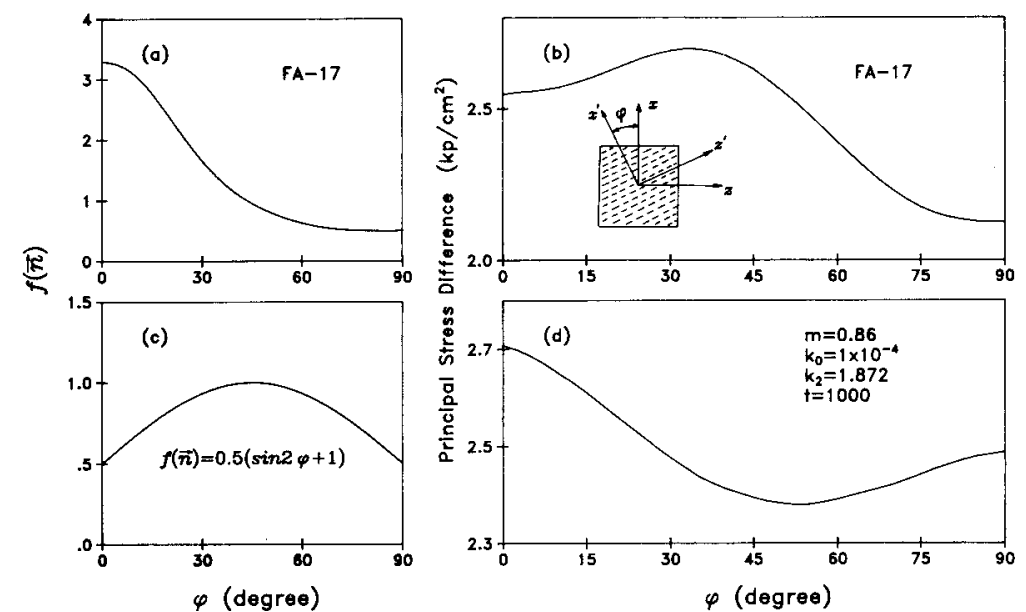

FIG. 9.-Principal Stress Difference Calculated for Specimens Cut Obliquely with Regard to Consolidation Direction 
from Ref. 1, 8, 9, and 18. The data set in Fig. 5 also involves two kinds of specimen as mentioned before. In the case of isotropic clay, the frequency distribution function $f(\overrightarrow{\mathbf{n}})^{\alpha}$ is equal to 1 for all directions.

Fig. 7 shows test data from the field vane test (23), for which, however, the frequency distribution function was not reported. Therefore, a uniform frequency distribution function was assumed in data fitting.

The curves in Figs. 4-7 represent creep isochrones, i.e., the curves connecting data points reached at the same times. The loading duration for Figs. 4-5 was 1,000 min, and for Figs. 6-7 it was 1 min.

Fig. 8 shows that the creep strain at constant stress evolves as a power function of time. Prior to starting the calculation with the aforementioned computer program, the value of exponent $m$ was determined for each data from regression plots exemplified the sample FA-17 in Fig. 8 The values of $m$, as well as parameters $k_{0}$ and $k_{2}$ obtained for optimum fits of individual data sets, are listed in Table 1.

Fig. 9 shows calculation results for the case when the cylindrical specimen is trimmed at inclination $\phi$ from a horizontally consolidated clay specimen. In this case the calculations must be done using the full $(6 \times$ 6) viscosity matrix and the full $(6 \times 1)$ stress and strain column matrices (whereas in the previous cases the calculations can be done using only $3 \times 3$ and $3 \times 1$ matrices, excluding all shear components).

It is interesting that the stress difference as a function of inclination

\section{TABLE 1.-Optimum Material Parameters (KInematically Constrained Microplane}

\section{Model)}

\begin{tabular}{l|c|c|c}
\hline \multicolumn{1}{c|}{$\begin{array}{c}\text { Test data } \\
(1)\end{array}$} & $\begin{array}{c}\text { Time } \\
\text { exponent, } m \\
(2)\end{array}$ & $\begin{array}{c}\text { Stress level } \\
\text { parameter, } k_{2} \\
(3)\end{array}$ & $\begin{array}{c}\text { Creep rate } \\
\text { parameter, } k_{0} \\
(4)\end{array}$ \\
\hline Bažant, Krizek, Ozaydin & & & \\
DA-2 & 0.77 & 4.587 & $0.40 \times 10^{-4}$ \\
FA-2 & 0.72 & 8.796 & $0.09 \times 10^{-4}$ \\
DA-17 & 1.00 & 2.412 & $0.69 \times 10^{-4}$ \\
FA-17 & 0.86 & 1.872 & $1.00 \times 10^{-4}$ \\
DI-2 & 0.82 & 5.403 & $0.45 \times 10^{-4}$ \\
FI-2 & 0.80 & 4.398 & $0.55 \times 10^{-4}$ \\
DI-17 & 1.00 & 2.223 & $1.50 \times 10^{-4}$ \\
FI-17 & 0.90 & 1.785 & $1.40 \times 10^{-4}$ \\
Murayma, Shibata & 0.99 & 8.169 & $0.9 \times 10^{-4}$ \\
Singh, Mitchell & 0.83 & 7.164 & $0.5 \times 10^{-4}$ \\
Campanella 1 & 0.86 & 3.771 & $0.5 \times 10^{-4}$ \\
Campanella 2 & 0.86 & 3.771 & $0.5 \times 10^{-4}$ \\
Campanella, Vaid & 0.61 & 19.101 & $0.5 \times 10^{-4}$ \\
Arulandan, Shen, Young & 0.80 & 2.952 & $0.26 \times 10^{-4}$ \\
SKA-EDEBY $\left(30 \mathrm{~cm}, T_{5}\right)$ & 0.88 & 8.169 & $0.5 \times 10^{-3}$ \\
SKA-EDEBY $\left(30 \mathrm{~cm}, T_{10}\right)$ & 0.89 & 6.534 & $0.83 \times 10^{-3}$ \\
KALIX (30 cm, $\left.T_{210}\right)$ & 0.84 & 6.159 & $2.5 \times 10^{-3}$ \\
SKA-EDEBY $\left(18 \mathrm{~cm}, T_{250}\right)$ & 0.73 & 11.184 & $0.4 \times 10^{-3}$ \\
\hline
\end{tabular}

${ }^{\mathrm{a}}$ They report only average strain rate, i.e., current strain divided by current time. The actual instantaneous rate is smaller, and this is why $k_{0}$ comes out so high from these data. angle $\phi$ first increases and then decreases [Fig. 9(b)]. However, this response is obtained only for certain orientation distribution functions $f(\overrightarrow{\mathbf{n}})$, e.g., the one measured (Fig. 2) and used in Figs. 4-7. For a nonmonotonic function $f(\overrightarrow{\mathbf{n}})$ such as that shown in Fig. $9(c)$, a different stressdifference dependence, first a decrease and then an increase, is obtained [see Fig. $9(d)$ ].

In regard to the curves in Figs. $9(b$ and $d)$ it may be observed that the dominant contribution to response stiffness (high stress difference) comes from the slipping on the platelets forming a $45^{\circ}$ angle with the specimen axis (because the normal deformations at interparticle contacts are considered zero). Thus, the orientation frequency distributions which peak at $\phi=45^{\circ}$ [Fig. 9(c)] give a decreasing stress difference between 0 and $45^{\circ}$, and those which peak at $\phi=0$ (the usual case, as considered here), give the opposite response, including an initial rise in stiffness at increasing $\phi[$ Fig. $9(b)]$, which at first seems suspect.

No comparison is made in Fig. 9 with the results of a certain test for inclined loading (8) shown in Fig. 4 (sample FA-17); such tests are scant and very difficult to perform, since it is impossible to realize homogeneous stress and strain boundary conditions without bulging and warping of the specimen. (The experimentalists who carried out this test have themselves expressed doubts about its reliability and relevance for the present study.)

The material parameters in the present model depend on the void ratio of the clay, the chemistry of the pore fluid, and the consolidation stress path. These dependencies appear to be similar as determined previously (8).

\section{Conclusions}

1. The current viscosity matrix of anisotropic clay can be calculated from the principle of virtual work based on the assumption that the shear strain rate that describes interparticle sliding along a contact plane (microplane) of any orientation is the resolved component of the macroscopic strain rate tensor.

2. In conformity with previous studies, the relation between shear stresses and shear strain rates on the contact planes (microplanes) may be based on the activation energy theory (rate process theory), which governs the dependence of creep rate on the stresses as well as temperature. In contrast to previous formulations except Ref. 8, the shear stress level used in the activation energy relation is not the same for all contact plane orientations, but varies with the orientation.

3. Material anisotropy may be characterized by the frequency distribution function for clay platelet orientations, which may be determined by direct observations (e.g., X-ray scattering).

4. The present micromechanics-based constitutive model is three-dimensional, improving on the previous model from Ref. 8 , which is limited to two dimensions. Also, the present model does not contradict the notion that clay platelets form fixed groups and sliding takes place only between neighboring groups, while the previous model from Ref. 8 implied that every particle was sliding. Although the model of the clay microstructure used in the present formulation is still rather simplified, it 
is considerably more refined than in previous works and may serve as a basis for further refinements, among which the inclusion of volume dilatancy will be needed most.

5. The present microplane model, which involves only two material parameters for the stress dependence and one for the time decay of creep rate, can be satisfactorily fitted to the existing data on the directional variations in the creep rate of remolded clay samples anisotropically consolidated in the laboratory. However, applicability in field situations to natural clay deposits remains experimentally unproven (such deposits, e.g., need not have a single particle fabric, which is not described by our model).

\section{ACKNOWLEDGMENTS}

Grateful appreciation is due to the US National Science Foundation for supporting this research under Grant No. CEE8211642 to Northwestern Univ.

\section{Appendix.-References}

1. Arulanandan, K., Shen, C. K., and Young, R. B., "Undrained Creep Behaviour of a Coastal Organic Silty Clay," Geotechnique, Vol. 21, No. 4, 1971, pp.

2. Baker, D. W., Wenk, A. R., and Christie, J. M., "X-Ray Analysis of Preferred Orientation in Fine Grained Quartz Aggregates," Journal of Geology. Vol. 77, 1969, pp. 144-172.

3. Batdorf, S. B., and Budianski, B., "A Mathematical Theory of Plasticity Based on the Concept of Slip," National Advisory Committee for Aeronautics (N.A.C.A.) Technical Note No. 1871, Washington, DC, Apr., 1949.

4. Bažant, Z. P."' "Microplane Model for Strain-Controlled Inelastic Behavior," Chapter 3, "Mechanics of Engineering Materials," C. S. Desai and R. H. Gallagher, Eds., John Wiley \& Sons, New York, NY, 1984, pp. 45-59.

5. Bažant, Z. P., and Oh, B. H., "Microplane Model for Progressive Fracture of Concrete and Rock," Journal of Engineering Mechanics, ASCE, Vol. 3, No. 4, Apr., 1985, pp. 559-582.

6. Bažant, Z. P., and Oh, B. H., "Efficient Numerical Integration on the Surface of a Sphere," Zeitschrift für Angewandte Mathematik und Mechanik (ZAMM),

7. Berlin, Germany, Vol. 66, No. 1, p. 37 .
Bažant, Z. P., and Oh, B. H., "Microplane Model for Fracture Analysis of Concrete Structures," Proceedings, Symposium on the Interaction of Nonnuclear Munitions with Structures," US Air Force Academy, Colorado Springs, CO, May, 1983, pp. 49-55.

8. Bažant, Z. P., Ozaydin, K., and Krizek, R. J., "Micromechanics Model for Creep of Anisotropic Clay," Journal of the Engineering Mechanics Division, ASCE Vol. 101, No. EM1, Feb., 1975, pp. 57-78.

9. Campanella, R. G., and Vaid, Y. P., "Triaxial and Plane Strain Creep Rupture of an Undisturbed Clay," Canadian Geotechnical Journal, Vol. 11, No. 1 , Feb., 1974, pp. 1-10.

10. Christensen, R. W., and Wu, T. H., "Analysis of Clay Deformation as a Rate Process," Journal of the Soil Mechanics and Foundations Division, ASCE, Vol. 90, No. SM6, Proc. Paper 4147, Nov., 1964, pp. 125-127.

11. Cottrell, A. H., The Mechanical Properties of Matter, John Wiley \& Sons, Inc., New York, NY, 1964

12. Diamond, S., "Pore Size Distribution in Clays," Clays and Clay Minerals, Vol. 18,1970 , pp. 7-23.

13. Edil, T. B., and Krizek, R. J., "Preparation of Isotropically Consolidated Clay
Samples with Random Fabrics," Journal of Testing and Evaluation, American Society for Testing and Materials, Vol. 5, No. 5, 1977, pp. 406-412.

14. Glasstone, S., Laidler, K. J., and Eyring, H., The Theory of Rate Processes, McGraw-Hill Book Co., Inc., New York, NY, 1941.

15. Krizek, R. J., Chawla, K. S., and Edil, T. B., "Directional Creep Response of Anisotropic Clays," Geotechnique, Vol. 27, No. 1, Mar., 1977, pp. 37-51.

16. Krizek R. J. Edil, T. B., and Ozaydin, I. K., "Preparation and Identification of Clay Samples with Controlled Fabric," Engineering Geology, 1975, Vol. 9, pp. 13-38.

17. Mitchell, J. K., "Shearing Resistance of Soils as a Rate Process," Journal of the Soil Mechanics and Foundations Division, ASCE, Vol. 90, No. SM1, Proc. Paper 3773, Jan., 1964, pp. 29-61.

18. Mitchell, J. K., Campanella, R. G., and Singh, A., "Soil Creep as a Rate Process," Journal of the Soil Mechanics and Foundations Division, ASCE, Vol. 94, No. SM1, Proc. Paper 5751, Jan., 1968, pp. 231-253.

19. Murayama, S., and Shibata, T., "On the Rheological Character of Clay," Transactions, Japan Society of Civil Engineers, No. 40, pp. 1-31.

20. Murayama, S., and Shibata, T., "Rheological Properties of Clays," Proceedings, 5th International Congress on Soil Mechanics and Engineering Founings, 5th International Congress on Soil

21. Murayama S and Shibata, T. "Flow and Stress Relaxation of Clays (Rheology and Soil Mechanics)," Proceedings, Rheology and Soil Mechanics Symposium of the International Union of Theoretical and Applied Mechanics, Grenoble, France, Apr., 1964, pp. 99-129.

22. Pande, G. N., and Sharma, K. G., "Multi-Laminate Model of Clays-A Numerical Evaluation of the Influence of Rotation of the Principal Stress Axes," Report, Dept. of Civil Engineering, University College of Swansea, UK, 1982.

23. Schwab, E. F., and Broms, B. B., "Pressure-Settlement-Time Relationship by Screw Plate Tests in Situ," 9th International Conference on Soil Mechanics and Foundation Engineering, Vol. 1, Tokyo, Japan, 1977, pp. 281-288.

24. Sheeran, D. E., and Krizek, R. J., "Preparation of Homogeneous Soil Samples by Slurry Consolidation" Journal of Materials, American Society for Testing and Materials, Vol. 6, 1971, pp. 356-373.

25. Singh A and Mitchell, J. K. "General Stress-Strain-Time Function for Soils," Journal of the Soil Mechanics and Foundations Division, ASCE, Vol. 94, No. SM1, Proc. Paper 5728, Jan., 1968, pp. 21-26.

26. Stroud, A. H., Approximate Calculation of Multiple Integrals, Prentice Hall, Englewood Cliffs, NJ, 1971.

27. Tan, T. K., "Structure Mechanics of Clays," Academia Sinica, Soil Mechanics Laboratory, Institute of Civil Engineering and Architecture, Harbin, China, 1957.

28. Taylor, G. I., "Plastic Strain in Metals," Journal of the Institute of Metals, Vol. 62,1938 , pp. $307-324$.

29. Tullis, T. E., "Experimental Development of Preferred Orientation of Mica During Recrystallization," thesis presented to the Univ. of California, at Los Angeles, CA, in 1971, in partial fulfillment of the requirements for the degree of Doctor of Philosophy.

30. Vaid, Y. P., and Campanella, R. G., "Time-Dependent Behaviour of Undrained Clay," Journal of the Geotechnical Engineering Division, ASCE, Vol. 103, No. GT7, July, 1977, pp. 693-709.

31. Wu, T Y, Resendiz, D. and Nuekirchner, P. J., "Analysis of Consolidation by Rate Process Theory," Journal of the Soil Mechanics and Foundations Division, ASCE, Vol. 92, No. SM6, Nov., 1966, pp. 229-248.

32. Zienkiewicz, O. C., The Finite Element Method in Engineering Science, 3rd ed. McGraw Hill Book, Co., Inc., New York, NY, 1977. 